\title{
ABM WITH BEHAVIORAL BIAS AND APPLICATIONS IN SIMULATING CHINA STOCK MARKET
}

\author{
Guocheng Wang ${ }^{1}$ and Shiguo Zhang ${ }^{2}$ \\ ${ }^{1}$ Institute of Quantitative \& Technical Economics \\ Chinese Academy of Social Sciences \\ Beijing 100732 China \\ ${ }^{2}$ Graduate School of Chinese Academy of Social Sciences \\ Beijing Fangshan 102488 China \\ zhangsg_999@126.com
}

\begin{abstract}
${ }^{1}$ One of the most important advantage of ABM (Agent-Based Modeling) used in social and economic calculation simulation is that the critical behavioral characteristics of the micro agents can be deeply depicted by the approach. Why, what and how real behavior(s) should be incorporated into ABM and is it appropriate and effective to use ABM with HSCA collaboration and micro-macro link features for complex economy/finance analysis? Through deepening behavioral analysis and using computational experimental methods incorporating HS (Human Subject) into CA (Computational Agent), which is extended $\mathrm{ABM}$, based on the theory of behavioral finance and complexity science as well, we constructed a micro-macro integrated model with the key behavioral characteristics of investors as an experimental platform to cognize the conduction mechanism of complex capital market and typical phenomena in this paper, and illustrated briefly applied cases including the internal relations between impulsive behavior and the fluctuation of stock's, the asymmetric cognitive bias and volatility cluster, deflective peak and fat-tail of China stock market.
\end{abstract}

\section{Introduction}

The complexity and variability in modern economic activity, especially complicated investment decisions in the financial market, have attracted much attention. Agent-based modeling (ABM) has progressed strongly in the economic/financial research. one of the most important advantage of ABM (Agent-Based Modeling) used in social and economic calculation simulation is that the critical behavioral characteristics of the micro agents can be deeply depicted by the approach. There are more and more rising call and urgent demand from new analytical and cognitive perspective to explore complexity in contemporary economy and finance, for their increasingly complex practice, widening lag gap of modern financial theory, and poorer tools; on the other hand, it will be occurring and breaking out for saving potential energy that accelerates more supporting and more effective theory and tools, driven by the interests of people in economy and finance and promoted by the big-data technique and so on. Due to realistic demand, it forces us to study and reveal the mystery of actual econ-

\footnotetext{
${ }^{1}$ Research has been supported by National Basic Research Program of China (973 project)( 2012CB955802) and National Natural Science Foundation of China (Grant No. 71471177).
} 
omy/finance from the visual angle of real behaviors of investors, to use the experimental method with the combination of HS (Human Subject) and CA (Computational Agent) and advanced research tools $[1,15]$, to penetrate and understand the volatility of stock market based on individual investing behaviors, to strive to find the inner relationship between the real and key characteristics of behaviors for investors at the micro-level and stylized facts or amount anomalies at the macro-level, the threshold value and sensitivity of critical point(s), and structural evolutionary process and so on.

Behavioral economics (finance), fateful arising and vigorous development, focuses on the qualitative analysis of micro individual behaviors up to now [6], and is based on the time series analysis and quantitative finance or financial econometrics method that is taking the zero mean, I.I.D, stationary and martingale process as the premise, logical starting point and analytic conditions, and ignoring or concealing the heterogeneous characteristics of behaviors for individual investors; whereas these neglected differences maybe are just the root causes of the complexity of financial markets. In the basis of deepening behavioral analysis, it is straightforward and driving tendency and new scientific approach to build integrated models with micro (behavior)-macro (output) link for uncovering the complexity of actual finance (economy), such as from the CAPM to (S) BCAP (Stochastic Behavioral Asset Pricing Models) and DSGE etc., that is the typical representative and foreshowing of this kind of cases [21]. This paper aims at discussing and exploring key behavioral characteristics and conducting mechanism each other surrounding the bias focus and the center axes of the relationship between micro-investing behaviors and macro-stylized facts (anomalies) in the stock market.

This paper is organized as follows. The following section contains the extended cognitive bias model and gives a brief analysis of micro-structural behavior causes for the complexity of financial market; then the principle of computational experimental finance with the method and implemental process of incorporating HS into CA is summarized, a benchmark model of behavioral analysis with micro-macro link is built and basic testing rules are discussed in Section 3; some relative work and ap- plied cases in recent years as well as preliminary experience are given in Section 4. The final section contains concluding remarks.

\section{The Key behavioral Characteris- tics of Micro-agent and Causes of Financial Market Complexity}

Anormalies frequently occurring in stock market and financial activities, such as the volatility cluster, deflective peak and fat-tail, and drastic fluctuation, are boosting up risks and crisis that already have existed. A measure that could foresee and avoid risks and similar situations could be found only if the root cause of such complexity is perceived, moreover, the individual behavior, which is influenced by the cognitive level and learning ability, is the critical point to understand the complexity, not only "irrational" real investment behaviors can be caused by the cognitive biased, but also the error between the expected and actual results can be improved by learning ability.

\subsection{Enriching Cognitive Biases}

Cognitive bias, we think, is materially to reflect the uneven and asymmetric of investor's preferences and behavioral representations on the investment products, so it can be understood as cognitive bias for a lot of bounded rationality or "irrational" real investment behaviors. Such as policy-sensitive, preferred stocks from emotion and psychology, herding effect, calendric effects examples in behavioral finance. Therefore, it is a good starting point to understand and to reveal the complexity of financial markets by exploring the cognitive bias of investors.

\subsubsection{CH model}

Hierarchy is an internal intrinsic property in the development of nature and human society that conforms to the law of cognitive development. Hierarchy represents dynamic changes in human behavior; thus, its existence is very rational and necessary. The complexity of human behavior can be determined by applying hierarchy to social science.

The $\mathrm{CH}$ (Cognitive Hierarchy) model begins with 0 -step players whose strategies are randomized equally. $K$-step players $(k \geq 1)$ believe that all 
other players use only 0 to k-1 steps. Assuming that k-type beliefs $g_{k}(h)$ regarding the proportions of lower-step $\mathrm{h}$ types denote a normalized true distribution,

$$
\begin{gathered}
g_{k}(h)=f(h) / \sum_{l=0}^{k-1} f(l) \\
g_{k}(h)=0, \text { for } h \geq k
\end{gathered}
$$

Therefore, both "partially rational" expectations and $g_{k}(h)$ approaches $f(h)$ are obtained as $k$ increases. Expected payoffs are computed, and the best responses are selected. Camerer et al. state that $f(h)$ follows the Poisson distribution [4]; Shao has developed an investment game model based on $\mathrm{CH}$ under the framework of bounded rationality from an investor group perspective [20]. The $\mathrm{CH}$ model has been widely applied in economic studies and it is a feasible approach to study cognitive bias with $\mathrm{CH}$ model.

\subsubsection{CH behavioral analysis}

Different properties are reflected in the $\mathrm{CH}$ model in consideration of the various cognitive abilities possessed by individuals. Our model focuses on two influential factors: first, stock price is a very important factor that influences investor decisions; second, policy adjustments in country-region China's stock market are of great concern. Both factors differ based on investors; however, determining the factor that is more significant to Chinese investors is an interesting topic. The heterogeneity of individual investors generally results in stock market diversity. Heterogeneous behavior causes the so-called leverage effect; that is, changes in stock prices or adjustments in the stock market are negatively correlated with volatility. Stock market heterogeneity often results in asymmetric, non-stationary, and nonlinear reactions. Stock forecasting is known as an uncertain, nonlinear, and non-stationary time-series problem; thus, accurately predicting the market via traditional methods is difficult. ABM can therefore be used to address arcane problems. Generalizing real stock market behavior is necessary to characterize heterogeneity.

\subsubsection{Extended CH model}

The financial market is often considered the selforganization of groups of interactive, learning, and bounded-rational agents in ABM. Investor behavior parameters for empirical data can be determined, and heterogeneous behavior can be calibrated using the given data. Hommes examines important stylized facts in financial time series by using simple economic and financial heterogeneous agent models [5].

Real macroeconomic phenomena can generally be described by Bayesian inferences based on linear and nonlinear behavior models. Integrated models are constructed based on human cognition, decision, and execution, and the transition from concrete individuals to the gross population is analyzed. We focus on endogenous weighted analysis and determine various behavioral attribute parameters in our model. The behavioral parameter $\lambda_{i}$ could be considered as the cognizing level in the benchmark model and can thus be used to describe stock market complexity as a whole. The cognitive parameter $\lambda_{i}$ can be influenced as follows: first, the differences among the natural instincts of individuals are significant; second, the responsiveness to external factors varies from individual to individual; third, reactions to interactions among individuals differ. Individual to economic $\mathrm{CH}$ is strongly related to previous macroeconomic phenomena.

However, cognitive biases, learning adaptability, and other the most frequent happening key characteristics are all owned by investors, so when the individual cognitive bias produced regarding the market, the ability that can modifies the prediction of the market according to cognitive biases which result from the divergence between the past prediction and past real market, this process can be called learning ability. Moreover, the behaviors of investors have static consistency and dynamic interactive adaptability in the learning process, Generally speaking, investors aim to avoid the results which result from the cognitive biases and achieve static consistency, which is also regarded as the desired aim for investors, in other words, the desired goal of investors is consistent with the real results. However, as the bounded rationality or "irrational" real investment behaviors would always exist for investors, the cognitive biases cannot be eliminated, then the process that the investors continuous eliminate the cognitive biases and aim to achieve the desired goals can be called dynamic interactive adaptability. Finally, the static consistency 
and dynamic interactive adaptability can describe manifestly the behavioral compatibility and recapitulating the complex behavioral relationship between individuals and financial market.

\subsection{Internal Relation between Complex Fi- nancial Market and Individual Behav- ior}

It is obvious that any phenomenon (result) occurred in the financial market is the interactions made by different individual investors and affected by many factors. The decision made by the individual investor changes as macro-policies, market signals and external impacts change, in the meantime, the financial market is also influenced by investors. Nevertheless, the behavioral compatibility means that the individual cognition, belief and strategy constantly adjust and change during the process; meanwhile, the proportion, structure, ways, track and process of evolution are synergistically changed as well. The behavior property, assets amount, response model to the external condition changed and differences in its intensity of an individual investor shall not be considered at many cases, nor these things are simplified to a random error or disturbance with mean value of zero. The investment behavior in Chinese capital market shall be considered more cautiously; otherwise it just copies other's behavior or falls into penny-wise misconceptions in thought.

The complexity in financial activities caused by individual subjective initiative (humanistic complexity) has some same parts with that of substances change in natural world but they have essential difference. Thus we need to further discuss the internal relation (a corresponding relation form could be built) between micro behavior characteristics and stylized facts. The individual behavior property, assets amount, opportunities and subjective wishes in humanity complexity are of significant difference and the causal chain among them is two-way, interdependent and interweaved with many factors and situations, which couldn't be speculated or reproduced according to some natural rules. However, some basic facts and objective attribute don't vary from person to person or from event to event (meet the individual irrelevance). For example, the whole economy/society is constituted by numerous individuals (dissoluble). The whole phenomenon is the individual behavioral outcome under the influence of individual behavior property and external conditions (constant relation). The behavioral process (rules), related behavior model, parameter, threshold and relational structure followed and presented by individual decisions could be perceived, simulated and reproduced.

This could be represented geometrically: the complicated financial market in reality is a compounded polyhedron. The theoretical mapping at any viewing angle and level is complicated and hard to define. The modern finance is a mapping system on a rational behavior section of the polyhedron built by an efficient market mechanism (see Figure 1); it also could spread to other behavior section (Bounded or non-rationality) with more feasible conduction and operation mechanism. Thus, a financial market of multiple dimensions could be obtained so as to find the internal relation between micro behavior and macro phenomena.

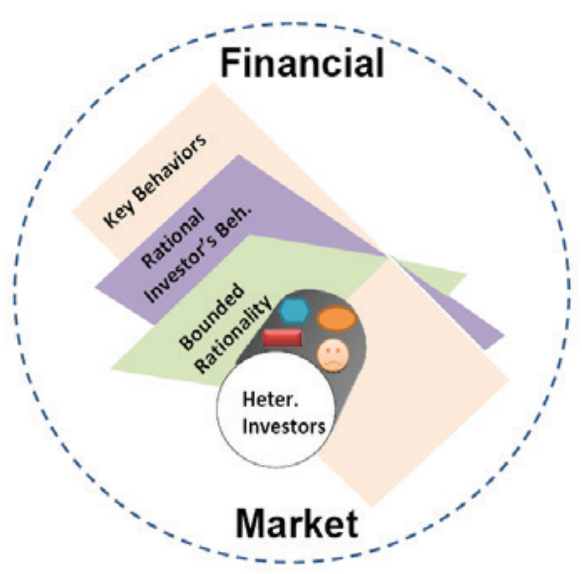

Figure 1. M-dimension behavioral sections for complex financial market

The relation between financial market outcome and investor behavior manifests such feature. Different individuals' investment behaviors are diverse as situation changes (depending on situation) as well as the response model and intensity. The path and influence of relations in economic network is asymmetric [7]. The conduction mechanism and evolution path are complicated and various. However, there is recognizable internal relation and needs material promotion of theory and method.

More focusing on the issue studied and promoting by scientific-technical progress, a synthetically 
analytical method that is people-oriented and covering all existed theories and methods is required and feasible. The influence of loss aversion, herd behavior, over-confidence, stock premium of outstanding bounded rationality (non-rationality) on stock market is hard to explain due to mixed affections of many factors and kinds of subjects by the traditional quantitative finance of modern financial theory based on the empirical approach. The behavioral finance couldn't be quantified with high cost and small coverage; the agent-based computational economics/finance (ACE/ACF) is in its initial stages [14], as well as some superior techniques like noise trading and wavelet analysis. Based on the above methods, all these methods lay a good foundation for independent and partial analysis but their limitation has been revealed day by day. It is better to set the investor's behavior as the logical starting point to analyze the theoretic root of financial complexity in reality. The conduction mechanism and evolution path from individual to group behavior are not single and changeless [16] so that a deepening, integrated model and coordinating analytical method are required. We try to construct theoretical analysis framework combined the static consistency and dynamic interactive adaptability to link the relationship between micro and macro based on the game equilibrium method [13].

\section{Agent-based Computational Fi- nance with incorporating HS into CA}

If we want to find the root cause and to understand furthermore the stock market, it is necessary to focus on the investors' behaviors and mutual relations (including response to rules, institutions and information). It's difficult to rationally portray and accurately quantify characteristics of real investing behavior. Comparatively speaking, the combination of HS and CA could both reflect the real investing behavior and be tested repeatedly under control [8]; it also breaks the limitation that the HS sample size is small and hard to represent the whole market as well as overcoming the substantial obstacle of pseudo-complexity ${ }^{2}$ produced by CA-based computational finance $[2,22]$.

\subsection{Basic Principle}

ACF with incorporating HS into CA is based on the changing character and rules of real behaviors made by investors and related agents in the modern financial activities. An experimental method that both follows scientific principle and reflects humanistic spirit and related financial complexity is applied to realize the integration of micro and macro, material and people (natural and social science), reductionism and holism (individual standard and relationship theory), human brain and computer (reality and virtual world, human wisdom and mechanical efficiency) as well as several model techniques. The evolutionary process could be reflected gradually; it also promotes the close relation between theory and reality so that they can verify each other.

\subsection{General Method and Steps}

Aimed at breaking the financial complexity, ACF with HS and CA could be divided into three steps according to modern finance, behavioral finance, complexity science and related knowledge:

1. Analysis of micro body behavior. The human behaviors in social activities can't be divided without linear transformation and similarity on the change of property and quantitative relation, and sometimes they are irreversible. What's more, the relation between individual and group rationality is complicated and its inconsistency may be the root of various social paradoxes. The driving forces, mode of action and conduction mechanism from individual to social action are complex. Increasing from individual to group amount is not only about quantity but also about the change of nature and direction $[9,10]$.

Seeing from the historical evolution of basic behavioral assumptions in economics, people have constantly been deepening and enriching their cognition on behavior property. The following figure shows the achievement and logical network of each stage, so that the developing path of investor's behavior could be compared.

By virtue of formalized language, the cognition depth of behavior could be classified and defined longitudinally to highlight the nature of behav-

\footnotetext{
${ }^{2}$ The computational complexity under given structural relationship is not real humanistic complexity; it is similar to the pseudorandom number generated by computer.
} 
ior. For a given capital market $E$ (including information and institutions), stock or investment goods set $X$, investor's set $N$, way and process of behavior $F$, investment behavior results set $Y$.

a. Deterministic type. $\forall x \in X$, for any $i \in N$, has the same choice behavioral way and process $f \in F$, necessarily $\exists y \in Y$ (only deterministic), makes $y=f(x)$.

b. Stochastic type. $\forall x \in X$, randomly select representative subject $i \in N$, has the same choice behavioral way $f \in F$, corresponding set of possible results $y_{x}=\left(y_{1}(x), \cdots, y_{m}(x)\right) \in Y$, accordingly, defined in $y_{x}$, there is a probability distribution $\Sigma_{x}$, the behavior result considered is expectation $E(x)=\sum_{j=1}^{m} f\left(y_{j}(x) \mid \Sigma_{x}\right)$; The general practice is that the digital characteristics of the sample substitute the ones of population in statistics.

c. Complex type. $\forall x \in X$, at the same time, consider all or several real subjects, the behavior pattern of each subject $i \in N$ (including the endogenous individual behavior property and the overall situation) is $f_{i}\left(s_{i}(x), s_{-i}(x), \lambda_{i}, X\right), s_{i}(\cdot)$ is $i$ 's strategic behavior, $s_{-i}(x)$ is strategic behavior of other investors excepting $i, \lambda_{i}$ is individual behavior parameters, $X$ can be extended to a general term that can affect the external environmental conditions of the main decisionmaking behavio; using such a behavior description, obtain a group of (certain) structural equations according to the rules of conduct and relationships structure; finally, basing on interaction and strategic behavior, create or deduct the response functions $R_{i}\left(s_{i}(x) \mid s_{-i}(x)\right)$.

The first two types of behaviors select representative bodies, implying a homogeneity assumption, namely all bodies only consider a behavior property and they follow the same decisionmaking strategy. Only one mechanism model from individual to group behavior is set. For the third type of complexity behavior, considering the difference and interaction among individuals, subjective consciousness and dependency on situation, the condition and interaction that permit multi-attribute and selectivity behavior could be described.
The assumptions of rational investors in modern finance theory revealed the limitation during the process of practice and challenge, so that the description on real investing behavior changes which needs observation, experiment, record, summarization, abstraction and classification. The essential attribute of human behavior could be perceived through behavioral expression. The micro behavior could be analyzed based on the experiment and two aspects of functions could be realized. One is to observe real response of human in various situations so as to further understand and describe behavior property; another is to find the way that how individual behavior comes to group behavior and how the overall characteristics of stoke market and economic activity affect. Without the support of modern computing technology, we only can rationally simplify the investment behavior and set an analytical model to find an optional solution.

The basic investment decision is nothing more than decision selection (buying, holding and selling) and the corresponding state could be recorded as $\{-1,0,1\}$. The totality and individual may form an alternative relation set so as to identify the basic model and build an analytical platform for theoretical framework. Different investors may make different decisions under different conditions. The highly abstracted rationality is an ideal not reality. Thus, we don't expect the theory established on such base could be understood better. Its main function is to offer a reference system under ideal state. For the analysis of characteristics and types of real investment behavior, we shall know what individuals take what investment behaviors under what environments and conditions through which conduction mechanism and achieved what result. The key behaviors will be parameterized through endogenous method, which is the important base of modeling and computational experiments. Thus, we could further study deeply and detailedly the focusing crowds and the interesting investment goods for a given market.

2. Integrated modeling, namely the integration of modeling or several models. Its basic form, structure type and flexible coupling or bridging technology could be used to carry 


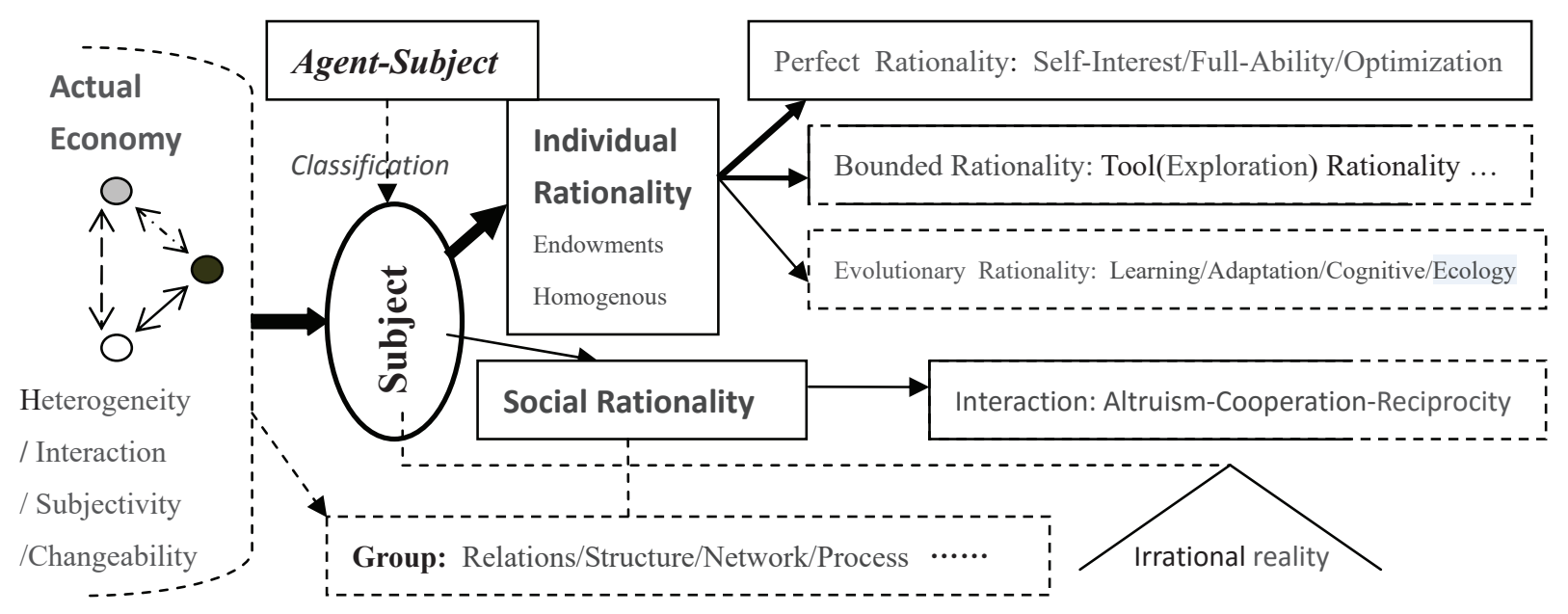

Figure 2. The spectrum of economic behavioral characteristics

out human-based (behavior) computational finance, which could organically integrate Quantitative Finance, Evolutionary Finance, Financial Engineering, Financial Mathematics, Financial Physics, and Financial Dynamics together in order to reveal the mystery of financial complexity.

The integrated model is the real response model and behavioral pattern of a vivid body in the form of scientific manner, establishing behavior rules, equation of relation and behavior (Dynamics). Based on the objective and overall analysis of subject behavior and centralized on the process of decision-making behavior, different models are established according to its constituent parts, stages and factors. Then each part and type of models is integrated through flexible coupling or bridging according to the natural process of realistic problems. Thus, such model is quite inclusive and flexible which could contain and connect existing models as per the need of problems and feasible conditions. It could selectively focuses on any special locality problem and integrates the distributed simulation and overall analytic method. For the specific topological structure and logical relationship, please see Figure 3.

The left side in this figure are the factors, policies that affect the investor's decision-making behavior and the right side is the decision result. Based on the integrated modeling of subject behavior, the overall process of decision making as well as the relation between individual investors and total amounts of stock market are revealed totally.

The meaning of soft-linkage or flexible coupling is the simulated mapping and links between parts of human and the nature and other complex relationships on the decision node. It is the join and verification between different models, namely bridging or coupling. For example, the output of previous step is translated into the input of next step; or changing name of variable, establishing similar instrumental variable, piecewise function of dummy variable in different forms; serving as the external environment parameter of subject behavior. Such connected models will stay the same as things change on the whole. By virtue of integrated model, the internal relation between micro and macro is revealed through construction of social function and related parameters adjusted in the steadystate process. The integrated modeling on the basis of subject behavior is a carrier or platform that calculates the social economy. The effectiveness, accuracy and sensitivity of the modeling could be evaluated by the actual degree of coincidence.

3. Computational experiments. The extreme assumption and implicated premise in the modern finance's empirical measurement couldn't summarize humanity features, such as human's experiences, intuition, comprehensive analysis, judgment and response. When the difference in risk preference, gaming instinct, price sensibility, interacting decision-making behavior, divi- 


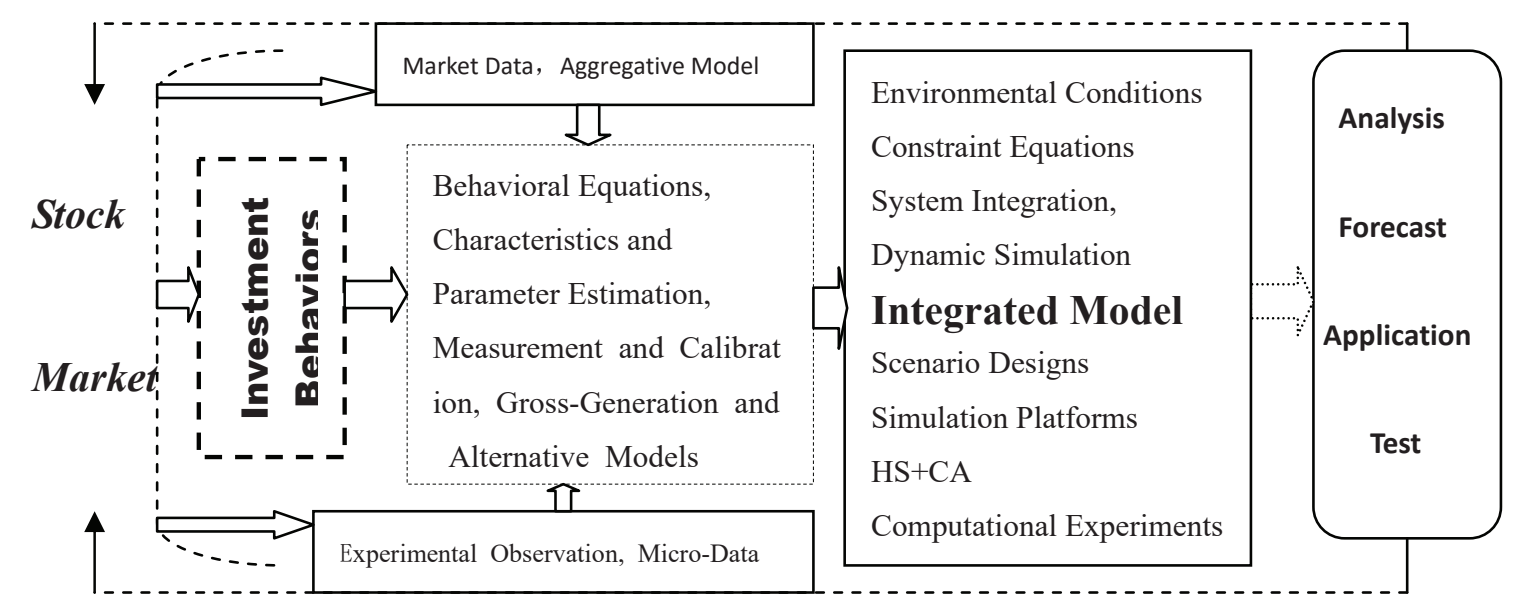

Figure 3. The flow chart of integrated modeling based on agent's behavior

sion or cluster of different investors group can't be ignored, data of actual investor's behavior type shall be gathered and screened, setting the original value of controlled variable, distribution pattern and range of variation. ${ }^{3}$ The data are input into the computer virtual subject as well as integrated model and computational experiment platform. By virtue of software such as MatLab, NetLogo, and genetic or bionic algorithm and comparing different scenarios, conditions and experiments, the micro behavior that causes market anomalies or stylized facts could be speculated by the output results. Various behavior pattern occurred in reality and conduction mechanism are sorted to calibrate and determine the key behavior parameters, set models, foresee potential market phenomena as the basis of investment decision.

The practicing experimental process is as follow: selecting all elements and possible corresponding combination (theoretically practical) among market scenario, behavior property, alternative mechanism, external condition and stylized facts setting. The conduction chain related to the internal link is calibrated and sorted according to generalized Bayesian Decision Theory so as to obtain a proper, real and scientific conclusion of persuasion and explanation power as well as brief and formal computational experiment result. Thus, the internal relation between individual investment behavior and market styl- ized facts or anomalies in the real capital market and the function relationship corresponding to the mechanism of evolving from individual to total amount are found. For the design and implementation of the experiment, results analysis, improvement and other necessary routine work, please refer to related content of experimental economics, finance and ACF.

\section{Benchmark Model and Behav- ioral Test}

Cognition also includes the agent's perception and judgment on the environmental conditions and gross change, it is natural and better to need the support of the micro-macro integrated model. This part is applied in the reference model of capital market with micro-macro link. It improves the integration of former research results $[17,18,19]$ and basic representation as well as micro behavior, and then discusses how to test and to analyze deeply the classical behavioral hypothesis.

\subsection{Benchmark Model}

Just considering a given stock market in China, let $y_{t}$ express net the output gap in period $t, r_{t}$ be the nominal interest rate, and $\pi_{t}$ be the rate of inflation, then we can obtain a series of behavioral equations of depicting macro-states with variables and relationship of between factors as follows:

\footnotetext{
${ }^{3}$ Practicing process is similar to the field-experiment; how to select samples, in-time observing and tracking in investors, and how to depict and grasp main characteristics during applications, will be seen in the further paper.
} 
Aggregate demand equation

$y_{t}=a_{1} \tilde{E}_{t}^{0}\left[y_{t+1}\right]+\left(1-a_{1}\right) y_{t-1}-a_{2}\left(r_{t}-\tilde{E}_{t}^{0}\left[\pi_{t+1}\right]+\varsigma_{t}\right)+u_{t}$

Aggregate supply equation

$$
\pi_{t}=b_{1} \tilde{E}_{t}^{0}\left[\pi_{t+1}\right]+\left(1-b_{1}\right) \pi_{t-1}+b_{2} y_{t}+v_{t}
$$

and the market behavior following the amending CityplaceTaylor's rules

$r_{t}=c_{1} r_{t-1}+\left(1-c_{1}\right)\left[c_{2}\left(\pi_{t}-\pi_{t}^{*}\right)+c_{3} y_{t}+c_{4}^{\mathrm{T}} \chi_{t}\right]+w_{t}$

Where: $\tilde{E}_{t}^{0}$ represents the expectation of the overall market (pseudo-player) at $t$ time, $a_{\bullet}, b_{\bullet}$ and $c_{\bullet}$ are factors or parameters to be estimated; $\pi *$ is the expected control targets of the increase; $\chi_{t}$ is a vector, which include all other factors impacting the yield, $\zeta$ is the risk and risk-free real interest rate spread; $u_{t}, v_{t}$ and $w_{t}$ are (random) disturbance/error term or white noise disturbance term.

The parameters could be obtained from the market empirical date according to traditional measuring empirical approach (related to the two fundamental assumptions, rational investor and EMH in modern financial theory), or be inferred from the investor's actual behavior in the market when there is Bias. Observing the actual investment behavior occurred in the stock market, an integrated model will be formed through construction of related behavior equation and connection with macro state equation, and then the computational experiment will be conducted to find out the potential correspondence between micro behavior and stylized facts (anomalies) in the stock market (see Figure 5). For the perfectly competitive market of general goods, the general equilibrium is the exception of game equilibrium under the condition of materialization. At this point, neutral system, complete information, individual rationality are satisfied with agreement of individual and collective rationality; if the assumption of perfectly competitive market is significantly deviated, a similar method could be used to find the internal relation between actual micro behavior and abnormal complex phenomena in the market. Thus, the complex economic and financial problems now could be better explained to find a way of quantifying evidences and realized the technology. The description and extension of micro investment behavior are as follows:

$$
I_{B}(i) \rightarrow \begin{cases}S_{b} & \text { at Prob. } p_{1} \\ S_{h} & \text { at Prob. } 1-p_{1}-p_{2} \\ S_{S} & \text { at Prob. } p_{2}\end{cases}
$$<smiles>CC=CC</smiles>

$$
I_{B}(i) \rightarrow \begin{cases}S_{b} & \text { at Thres. Value } T B_{b} \\ S_{h} & \text { at Thres. Value } T B_{h} \\ S_{s} & \text { at Thres. Value } T B_{s}\end{cases}
$$

The behavior property, critical change and threshold difference reflect individual diversity (heterogeneity). The determination of threshold connects the combined action of numerous agents' behavior and external conditions change, including the influence among agents, namely endogenesis and interaction; the threshold is determined according to the total available market received by the individual, other investors' strategies and individual characteristic parameters. It could also be used in various key behavior characteristics for the agents in general market.

\subsection{Hypothesis Testing of Basic Behavior}

The behavior, structure and output (variable, equation and parameters etc.) are integrated in the above equation and description. The change of macro aggregate in the specific application is expressed as actual situation and its key point is to determine the basic attribute and type. For the estimation of parameters, macro equation is still a common method for econometrics and its main change is that the data sources are based on the macro abnormal situations and related data and integration method. The micro behavior parameters are obtained from the personalized features from agent's behavior through endogenalization and game experimental method. The hypothesis of basic behavior could be gradually released and expanded to make up the logical losses by key features among complete rationality, limited rationality and actual behaviors. The inspection of basic behavior hypothesis is divided into two directions and ways.

1. Prior Testing. To suppose null hypothesis or zero hypothesis $H_{0}$ : (limited) rational investment behavior, the total investment behaviors in the market are sampled following the given test 
criterion. Make an accepted or rejected decision according to the distribution function structure statistics and judging standard, namely the theory that investor's behavior is (limited) rational is accepted in the actual analysis. The derivative of related hypothesis has the same basic attribute, way and structure of organizing groups and dynamic procedure.

2. Posterior Testing. Setting first a set of assumptions $H=\left\{H_{0}, H_{1}, \cdots, H_{N}\right\}$ that represent the $\mathrm{N}$ kinds of possible behaviors according to different typical facts, following then Bayesian criterion of decision and likelihood principle, by the forward, choosing that the most likely causes stock market behavior factor $H_{i 0} \in H$ of typical facts (events) $E_{0}$, to meet $P\left\{H_{i 0} \mid E_{0}\right\}$ $\geq \underset{H_{i} \in H}{\operatorname{Max}}\left\{P\left\{H_{i} \mid E_{0}\right\}\right\}$. By so testing, it also reflects the macro-micro link, interaction, mutually causes and effects each other. Especially under the conditions of different economic environment and subject's behaviors, it should be more rational in hypothesis testing, making up the lack of logic to lay a solid foundation for scientific and theoretical guarantee; otherwise the obtained theoretic results and analytic conclusions can only be built on the sand castle and the product of thinking with penny-wise. As for the combination of complex behavior, it should exist $\Sigma *=\left(\omega_{1}, \omega_{2}, \cdots, \omega_{l}\right)$ to make

$$
p\left\{\Sigma * H_{l} / E_{0}\right\} \geq p\left\{\sum_{N^{\prime}} * H_{l} / E_{0}\right\}, \forall N^{\prime} \subseteq N
$$

This is actually a holistic related inspection presumption estimation method of parameters. Of course, it is also caused by synthetically considering and contains the synergistic effect, the structure and the total amount generated in the process of change.

\section{Applications}

Combining with the research on Chinese stock market quotation and investors' behavior, we want to find what kinds of behaviors correspond to the stylized facts and anomalies, taking Shanghai Stock Exchange's actual data as example and using cur- rent financial database and professional software to carry out tentative application. We choose parts of cases for positive supporting. ${ }^{4}$

\subsection{Investment Impulsion and Share Price Change}

Based on the actual investment behavior of Chinese investors and taking 254 actual historical data from Jan.4, 2007 to Jan.18, 2008 as example. The types of behaviors are analyzed as the original value, using Matlab software to simulate. Take 1 business day (or unity of time) as step length and 500 business days as the total length of the simulation. Firstly, the static simulation on the basic model constructed by comparing investors' normal behaviors is studied to obtain the result of basic model analysis. Then, considering the dynamic evolution of various groups, we focus on the investment impulsion behaviors. Paying attention to the learning adaptability, difference and interaction of investor's investment decision-making and supposing other factors that influence behaviors unchanged, simplification or discard will be made (according to requirements and increasing or changing behaviors characteristics and influencing factors).

Specific method: the reaction speed (change the time of decision rule lasting) and strength (the proportion of individual trading volume in its total assets) are used to describe the individual investment impulsion behavior; when the external information or market trend changed, the trade that decision rule changed is called as first-class impulsion behavior; the investment behavior that is changed two days or more later is called as second-class impulsion behavior. These two kinds of behaviors affect and transmute into each other. Taking the max daily fluctuations of stock prices $8 \%$ as critical value, the fluctuating value (mental threshold) mentally bore by the individual is recorded as $w$, deciding $\mathrm{w}$ and different impulsion behavior and taking $5 \%$ as the contrast threshold; when first-class (second-class) behavior dominates, the stock market will boom (slump). The time of duration for these two behaviors before the stock market changes is quite different (see Figure 4); the length of variable section near the threshold is subdivided and narrowed to observe the sensibility and critical effect of in-

\footnotetext{
${ }^{4}$ The author gratefully acknowledge Dr. Fei Liu and Dr. Yuntao Long for conducting examples, seeing their dissertation (Liu, 2009; Long, 2013) respectively.
} 
vestor's behaviors. If the investor could make decision before the critical point of market fluctuation, the earnings are considerable. The new findings in this researching application are as follows:

1. The influence and relation between the investment behavior characteristics and price fluctuation in the stock market are analyzed from the micro-view; comparatively speaking, individual investment impulsion behavior is internally related to the stock market booming (slumping); the first-class behavior may cause booming and the second-class behavior may cause slumping;

2. Trying to explore the multi-dimension description and classified quantitative method of micro motivation and to use investors' actual behavior to study the investment impulsion behavior;

3. The computational experiment is used to explore the micro-cause of stock market complexity in country-region China, trying to reveal the mechanism of overall complex phenomena caused by the potential individual behavior model. The main performance of investor's mental fragility is sensibility around the critical point(s).

What's more, from the point of methodology, acceptance or adjustment of rational behavior assumption, establishment of causality or theoretical logic model and utilization of empirical data could not fit the need of actual complexity. However, micro-behavior-based computational experiment analysis focuses on the actual investment behavior of different agents and development of group structure, simulating different results of convergence, violent shock, booming or slumping. It shows the necessity to further study different agents' inter-behaviors characteristics. This method could be one of the best ways to discover and perceive the complex essence of abnormal economy and financial activities.

\subsection{Type of Information Preference and Market Sensitiveness}

The developing stock market in China may prefer any kind of information. General quantitative finance and statistic testing may result in large errors due to lack of inspection of behavior basic hypothesis. We use computational experimental finance to combine HS and CA based on the investor's actual behavior and real data of the stock market. By comparing different responses by different investors to various policies and market signals, policy sensitivity parameters and tendency parameters influenced by the vicinity are designed, taking proportions of different investors as controlled variable in order to observe the different preference type and market effect identified and responded by the investor's behavior biases and market structure change to the policy and market signals; these were further parameterized and changed according to the potential situations to know whether the numerous investors' behaviors coincide with the stylized facts occurred in reality, focusing on internal relation between cognitive behavior and gathered fluctuation. The specific simulation has been launched in NetLogo 5.0.3 version.

According $\mathrm{CH}$ model above, we let $h_{1}, h_{2}$ respectively express signal or information from market foundation and policy, $f_{1}\left(h_{i}\right), f_{2}\left(h_{i}\right)$ represent institutional and individual cognitive preference weights on $\left\{h_{1} h_{2}\right\}$. It can be reached the conclusion that $f_{\bullet}\left(h_{1}\right)<f_{\bullet}\left(h_{2}\right)$ and $f_{2}\left(h_{2}\right)>f_{1}\left(h_{2}\right)$ through computational experiment. Therefore we can infer that Chinese stock market is more policy-sensitivity along with individual investor is more sensitive than institution.

We find that the investor quite depends on the policy effect by comparing and policy sensibility is relatively general conclusion. If we further study what kind of external information and impact that Chinese investors are sensitive to, an effective differentiation and coping strategy on investment will be obtained. The actual effects of policies are closely related to the investor's expectation on policies and market sentiment. At first, there is a good policy or news, but if it quite differs from what the investor expects, the actual effect in the stock market will become bad (for example, the effect caused by interest adjustment for several times). The institutional investors and private investors have different ways of response and sensibility to the market signal and policy information due to different preference on information and proportion structure. However, we can't conclude that Chinese stock market is an efficient, semi-efficient or weak-efficient market nor summarize this market as policy-dependent stock market. Our aim is to let the policy, stock mar- 


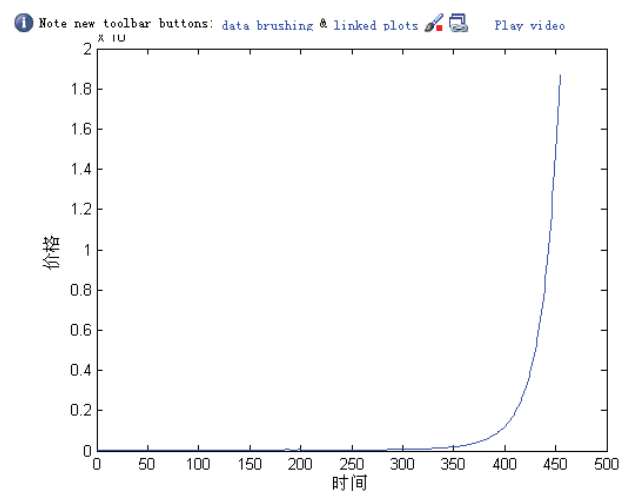

$W>8 \%\left(\right.$ the $1^{\text {st }}$ impulsive behaviors $)$

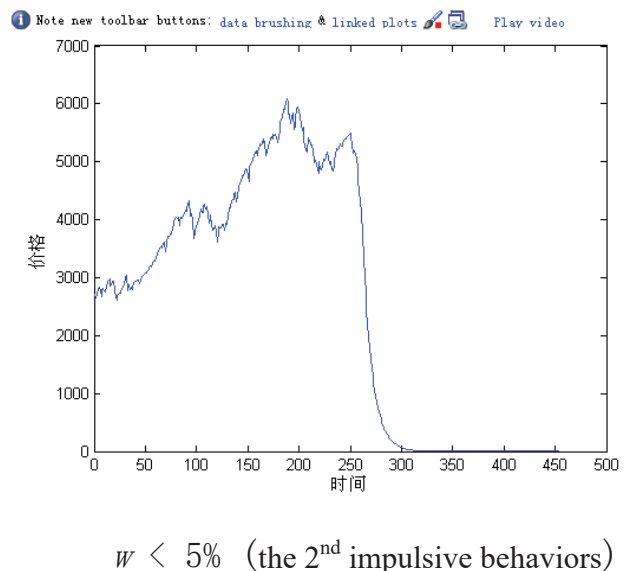

Figure 4. The relation simulation between different types of impulsive behaviors and sharp stock-price. Note: Vertical axis is price; Horizontal axis is time

ket and investor achieve their expected goals by adjusting investment strategy under the effect of policy; meanwhile, the policy launched has to be more targeted in good time, then it could achieve the expected effect; it's not appropriate to conclude the advantage and disadvantage of a policy and the policy responding type of the stock market $[11,12]$.

\subsection{The Essence of Behavior and Integra- tive Effect of the Market}

The asymmetry on benefits and losses in people's behavior is inherent. Several behavior-bias and their combination of key behavioral characteristics illustrated in the behavioral finance could be the main reason that induces some stylized facts or anomalies in the market (see Figure 5). The key problem to research in our work is that what reasons and approaches could convincingly prove it.

Asymmetric investment behavior (key behavior characteristic) has a complicate and internal relation with market anomalies (stylized facts), such as impulsive action vs. share price fluctuation, asymmetric cognition vs. fat-tail and drastic moves and stock market differentiation, herd behavior, psychological complex vs. blue-chip gathering, nonrational, irregular investment, overconfidence vs. stock market bubble, information preference and market awareness, overall features, individual response, behavior pattern vs. trend and development of the stock market. Observing and judging the situation, we could take measures before group behavior and market generate results or other investors find this, so as to decide our investment behavior. For the actual behavior of Chinese investors, they have to face the market complexity and stylized facts and integrate theories by changing ideas, which is well-suited and effective and also fit the essence of market volatility.

\section{Conclusions}

The modern finance undoubtedly is more and more complicated but it could be recognizable. Computational experimental finance with agent-based and integrated modeling is scientific, acceptable and effective. Viewing from investor's behavior, Chinese financial (stock) market has significant behavioral biases, presenting as policy sensibility (depending on conditions and structures) but irregularly responds to the market signal. Thus, the theoretical method based on rational investment and efficient markets hypothesis is prudently used to study Chinese problems. It's necessary to consider the adaptation of method aiming at solving problems.

The market effect of potential actual investment behaviors shall be further studied. Different investors shall determine their behavioral characteristics by themselves and choose a proper way of investing. The theoretical method in this paper aims at the stylized facts of capital market, realistic investor's behavior and economic environment in country-region China. It's worth deepening and promoting as it is constantly proof-testing.

\section{Acknowledgment}

We are grateful to the National Basic Research Program of China (973 Program) (Serial No. 


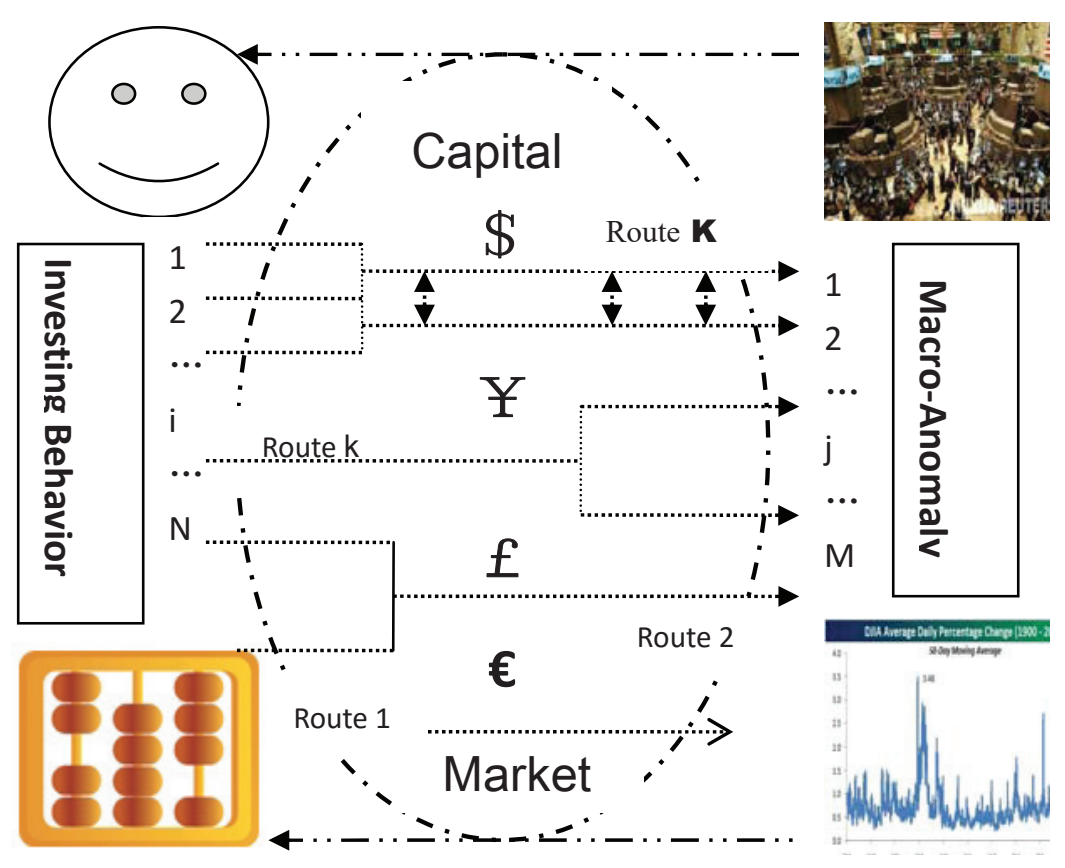

Figure 5. Mapping from Actual Investing Behavior to Macro-Anomaly in Stock Market

2012CB955802) and the National Natural Science Foundation of China (No. 71471177).

\section{References}

[1] B. Arthur, Economic Agents that Behave like Human Agents, Journal of Evolutionary Economics, Issue 3, 1993, 1-22. Reprinted in The Legacy of Joseph A. Schumpeter, H. Hanusch, Ed., Edward Elgar Publishers, 2000.

[2] B. LeBaron, Agent-based computational finance, In: Tesfatsion L., Judd K. L. (Eds.), Handbook of Computational Economics. Elsevier, 2006, 11871233.

[3] B. LeBaron, Heterogeneous gain learning and the dynamics of asset prices. Journal of Economic Behavior and Organization. Vol.83, $2012,424-445$.

[4] C. F. Camerer, T. Ho and J. K. Chong, A cognitive hierarchy model of games. Quarterly journal of economics, 119(3), 2004, 861—898.

[5] C. H. Hommes, Heterogeneous agent models in economics and finance. In L. Tesfatsion and K.L. Judd (Eds.), Handbook of computational economics, volume 2, 2006, Agent-based computational economics. Amsterdam, the Netherlands: Holland/Elsevier.

[6] D. Levin, Is Behavioral Economics Doomed? The Ordinary versus the Extraordinary. UK: openbook publishers, 2012.
[7] F. Schweitzer et al., Economic Networks: The New Challenges", Science, 24 July, Vol. 325. No. 5939, 2009, $422-425$.

[8] G. Wang, Exploring complex economy to develop quantitative economics from Micro-behavior perspective, Journal of Quantitative Economics, Vol. 2(1), 2011, 102-120.

[9] G. Wang, The evolvement and beyond of rationalism in modern economics. Social Sciences in China, No. 7, 2012, 66-82.

[10] G. Wang, Deepening micro-behavioral analysis and exploring the complexity of macro-economy. Jiangsu Social Sciences, No. 3, 2013, 20-28.

[11] G. Wang, Behavioral Macro-Financial Modeling from Investor's Bias with Applications - Based on the Experiment of Incorporating HS and CA, Journal of Management Science \& Statistical Decision Vol. 11, no.1, 2014, 24-40.

[12] G. Wang, and Y. Long, Study on Emergence of Capital Market with Cognitive Hierarchy and Extensive Agent-Based Modeling - An Application of e-Science in Social Sciences, e-Science Technology \& Application, 5(1), 2014, 83 92.

[13] G. Wang, and S.G. Zhang.,Behavioral Compatibility, ACF and the Emergence of Chinese Stock Market, 21st International Conference on Computing in Economics and Finance, Taipei, Taiwan, June 20-22, 2015.

[14] J. D. Farmer and D. Foley, The economy needs agent-based modeling, Nature, ( $6^{\text {th }}$ August) 460, 2009, 685-686. 
[15] J. Duffy, Agent-based models and human subject experiments. In: Tesfatsion L., Judd K.L. (Eds.),Handbook of Computational Economics, Volume 2, 2006, 949-1011. North-Holland, Amsterdam, the Netherlands: Elsevier.

[16] J. H. Miller and E. Scott, Complex Adaptive Systems: An introduction to computational models of social life, NJ: Princeton university press, 2007.

[17] L. Scheffknecht, and F. Geiger, A behavioral macroeconomic model with endogenous boombust cycles and leverage dynamcis, FZID Discussion Papers 37-2011, University of Hohenheim, Center for Research on Innovation and Services, 2011.

[18] M. Lengnick and H. W. Wohltmann, Agent-based financial markets and New Keynesian macroeconomics: a synthesis, Journal of Economic Interac- tion and Coordination, Springer, vol. 8(1), 2013, 1-32.

[19] P. De Grauwe, Top-Down versus Bottom-Up Macroeconomics, CESifo Economic Studies, 56, 2010 , 465-497.

[20] P. Shao, Bounded rationality, cognitive hierarchy and investment game. The journal of quantitative \& technical economics. 10, 2010, 145-155.

[21] T. Lux, Stochastic Behavioral Asset-Pricing Models and the Stylized Facts, In: Handbook of Financial Markets: Dynamics and Evolution. Edited by Thorsten Hens and Klaus R. Schenk-Hoppe, chapter 3, 2009, 161-215.

[22] W. Zhang, Y. J. Zhang and X. Xiong, Agent-based Computational Finance, Beijing, Science Press, 2010 .

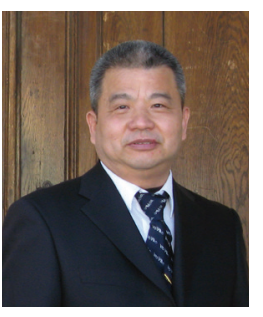

G.C. Wang researcher: the senior researcher who works at the Institute of Quantitative \& Technical Economics, Chinese Academy of Social Sciences, the standing member of Chinese Association of Quantitative Economics, the chairman of China Association of Game Theory and Experimental Economics, etc.

Academic Degree programs:

- B.A. in mathematics, China University of Geosciences, 1977 1982

- M.A. in mathematical geology, China University of Geosciences, 1985 1988

- Ph.D in Quantitative Economics, Graduate School of Chinese Academy of Social Sciences, 1993 1996

Research direction: game theory, behavioral and experimental economics, decision simulation for complex economy and finance, etc 